\title{
Thrombectomy in special populations: report of the Society of NeuroInterventional Surgery Standards and Guidelines Committee
}

\author{
Fawaz Al-Mufti, ${ }^{1}$ Clemens M Schirmer (1) ,' Robert M Starke, ${ }^{3}$ Neeraj Chaudhary, ${ }^{4}$ \\ Reade De Leacy, ${ }^{5}$ Stavropoula I Tjoumakaris (D) , ${ }^{6}$ Neil Haranhalli, ${ }^{7}$ \\ Isaac Josh Abecassis, ${ }^{8,9}$ Krishna Amuluru, $^{10}$ Ketan R Bulsara, ${ }^{11}$ Steven W Hetts (1) , ${ }^{12}$ \\ On behalf of the SNIS Standards and Guidelines Committee and SNIS Board of \\ Directors
}

\begin{abstract}
- Additional supplemental material is published online only. To view, please visit the journal online (http://dx.doi. org/10.1136/neurintsurg2021-017888)
\end{abstract}

For numbered affiliations see end of article.

Correspondence to Dr Steven W Hetts, Department of Radiology, UCSF, San Francisco, CA 94143, USA steven.hetts@ucsf.edu

Received 12 June 2021 Accepted 22 June 2021
Check for updates

C) Author(s) (or their employer(s)) 2021. No commercial re-use. See rights and permissions. Published by BMJ.

To cite: Al-Mufti $F_{\text {, }}$ Schirmer CM, Starke RM, et al. J Neurolntervent Surg Epub ahead of print: [please include Day Month Year]. doi:10.1136/

neurintsurg-2021-017888

\section{ABSTRACT}

Background The purpose of this guideline is to summarize the data available for performing mechanical thrombectomy (MT) for emergent large vessel occlusion (ELVO) stroke in special populations not typically included in large randomized controlled clinical trials, including children, the elderly, pregnant women, patients who have recently undergone surgery, and patients with thrombocytopenia, collagen vascular disorders, and endocarditis.

Methods We performed a literature review for studies examining the indications, efficacy, and outcomes for patients undergoing MT for ischemic stroke aged $<18$ years and $>80$ years, pregnant patients, patients who have recently undergone surgery, and those with thrombocytopenia, collagen vascular diseases, or endocarditis. We graded the quality of the evidence. Results MT can be effective for the treatment of ELVO in ischemic stroke for patients over age 80 years and under age 18 years, thrombocytopenic patients, pregnant patients, and patients with endocarditis. While outcomes are worse compared to younger patients and those with normal platelet counts (respectively), there is still a benefit in the elderly (in both mRS and mortality). Data are very limited for patients with collagen vascular diseases; although diagnostic cerebral angiography carries increased risks, MT may be appropriate in carefully selected patients in whom untreated ELVO would likely result in disabling or fatal outcome.

\section{RECOMMENDATIONS}

1. Mechanical thrombectomy (MT) should not be withheld from octogenarians and nonagenarians, but rather be evaluated on a case-by-case basis. (class IIa-b, level B-NR and C-LD)

2. MT should not be withheld from thrombocytopenic patients. (class IIb, level C-LD)

3. There is no absolute minimum platelet count that excludes patients from MT given the high morbidity of emergent large vessel occlusion (ELVO). The expert panel considers $<20 \mathrm{~K}$ very concerning for potential hemorrhagic conversion of infarction or procedural complication, and hematologic consultation to understand the underlying etiology of extreme thrombocytopenia is reasonable. Some experts also consider platelet transfusion to be reasonable in patients with very low platelet count. (class IIb, level C-EO)

4. MT should not be withheld from neonates, infants, children, and adolescents, but rather be evaluated on a case-by-case basis. (class II $a-b$, level B-NR and C-LD)

5. Standard techniques for radiation dose minimization (ALARA - as low as reasonably achievable) should be applied in diagnostic and therapeutic applications for pediatric stroke. (class I, level B-NR)

6. Where possible, and determined by local availability, initial radiologic work up of stroke in neonates, infants and children below the age of 10 should involve non-ionizing radiation, specifically MRI. (class IIa, level B-R)

7. Potential benefits and risks of MT should be evaluated on a case-by-case basis in patients with collagen vascular diseases with reference to the specific underlying disease. (class IIbIII, level C-EO)

8. Diagnostic catheter angiography in patients with collagen vascular disease carries increased risks; non-invasive imaging should be used whenever possible. (class III:Harm, level C-EO)

9. MT should not be withheld from patients who have recently undergone surgery. (class IIa, level C-LD)

10. MT should not be withheld from patients with endocarditis. (class IIb, level C-LD)

11. Endovascular thrombectomy is a safe and feasible procedure for treating acute ischemic stroke due to ELVO in pregnancy, and should be considered for patients in a similar manner as would be indicated in a non-pregnant patient. (class IIa, level C-LD)

12. Fluoroscopic radiation safety features should be fully used as well as a low threshold for anesthesia and obstetrics consultation in pregnant patients. (class IIb, level C-LD)

\section{INTRODUCTION}

The pendulum for the usefulness of mechanical thrombectomy (MT) for ischemic stroke treatment has gone from one extreme to another in recent years. Decades from now, 2015 will be remembered 
as a pivotal year in establishing it as the standard of care for large vessel occlusions. ${ }^{1-5}$ A pooled patient analysis of these trials concluded that MT in patients who met inclusion criteria led to significantly reduced disability compared with controls. ${ }^{6}$ For every 100 patients treated, 58 will have improved functional status compared with controls, and approximately 20 will achieve independence. ${ }^{6}$ As promising as the results have been in a relatively homogeneous population, the question of generalized applicability of these findings to a more heterogeneous population has been a topic of debate. ${ }^{67}$ Stroke systems of care predicated on these trials, as well as protocols to consider during the COVID-19 pandemic, have been extensively reviewed and incorporated into other standards and guidelines documents from the Society of NeuroInterventional Surgery ${ }^{8-18}$ The majority of patients treated with MT in real-life practice, would probably not have qualified for inclusion in the clinical trials, but probably do benefit from treatment. ${ }^{7}$

\section{GUIDELINE SPECIFICS: CURRENT GUIDELINES FOR PATIENTS WITH EMERGENT LARGE VESSEL OCCLUSION ACUTE ISCHEMIC STROKE}

Current US standards for the role of mechanical thrombectomy in emergent large vessel occlusion (ELVO) anterior circulation stroke are well summarized in the 2019 update to the 2018 guidelines for management of acute ischemic stroke published by the American Heart Association and American Stroke Association. ${ }^{19}$ Section 3.7 of that document includes guidelines on: (1) thrombectomy after intravenous thrombolysis, (2) thrombectomy 0 to 6 hours after last seen well, (3) thrombectomy 6 to 24 hours after last seen well, (4) anatomic targets, (5) thrombectomy techniques, (6) adjunctive antiplatelet and fibrinolytic medications, and (7) postprocedural blood pressure management. Table 1 below summarizes those recommendations as well as the class (strength) of recommendation and the level (quality) of evidence for each.

The American Heart Association (AHA)/American Stroke Association (ASA) guidelines are based on randomized controlled clinical trials such as DAWN, DEFUSE 3, ARISE 2, EXTEND-IA, REVASCAT, SWIFT-PRIME, ESCAPE, and MR CLEAN, all of which enrolled adult patients (18 years old or older) and some of which excluded very elderly patients (older than 80,85 , or 90 years in some trials, and also based on case series and expert opinion. In addition to extremes of age, other common criteria that have excluded patients from randomized trials are pregnancy, thrombocytopenia, active endocarditis, recent surgery, and collagen vascular disease. Patients who have one or more of these characteristics fall outside the mainstream of medical evidence to date and, thus, will be the focus of the remainder of this guideline document.

\section{METHODS}

We systematically reviewed the literature for manuscripts with the key word 'stroke' and any of the following: 'mechanical thrombectomy', 'elderly', 'octogenarian', 'nonagenarian', 'pediatric', 'pregnant', 'pregnancy', 'thrombocytopenia', 'low platelets', 'collagen vascular disease', 'connective tissue disorder', and 'endocarditis'. These topics were chosen to reflect conditions not typically included in randomized controlled trials of stroke that were already published in 2020 or expected to be published in 2021. We graded evidence using the Oxford Centre for Evidence-Based Medicine guidelines ${ }^{20}$ and the American Heart Association guidelines, ${ }^{19}$ with the latter being applied to recommendations.

\section{Octogenarians and nonagenarians}

The proportion of elderly ( $\geq 65$ years) individuals in the United States is expected to double by the year 2050, and people older than 80 years of age have the highest incidence of acute ischemic stroke. ${ }^{21}$ Thus, the proportion of patients aged $\geq 80$ years who are eligible for endovascular therapy (EVT) for acute ischemic stroke (AIS) will continue to increase. This patient population was excluded from three of the six pivotal MT trials. ${ }^{352}$ Furthermore, this population was relatively under-represented in the trials that did include them, suggesting possible selection bias. $^{21}$

Several retrospective and prospective observational studies have reported MT for elderly patients. The generalizability of these studies is limited by small sample sizes, restriction to single centers, significant heterogeneity, and often without comparison with optimal medical management. ${ }^{21}$ Meta-analysis data from the Highly Effective Reperfusion evaluated in Multiple Endovascular Stroke trials (HERMES) trial showed better 90-day outcomes among patients aged $>80$ years treated with thrombectomy than for patients treated with intravenous thrombolysis alone $(29.8 \%$ vs $13.9 \%$; common $\mathrm{OR}=3.68,95 \%$ CI 1.95 to 6.92). ${ }^{6}$ Additionally, elderly patients who underwent MT had a slightly reduced risk of death (28\% vs $45 \%$; adjusted rate ratio $0.60,95 \%$ CI 0.36 to 0.99 ).

Hilditch et al performed a systematic review of 16 observational studies involving patients aged $\geq 80$ years who underwent MT for AIS and combined this with data from the HERMES study. Among 860 patients, the authors showed a $27 \%$ rate of functional independence at 90 days (95\% CI 21\% to $32 \%$ ), and a mortality of $34 \%$ (95\% CI $23 \%$ to $44 \%$ ), with significant heterogeneity between published reports $\left(\mathrm{I}^{2}=70 \%\right.$ and $91 \%$, respectively). Although recanalization rates were excellent, $78 \%$ rate of Thrombolysis in Cerebral Infarction (TICI) $2 \mathrm{~b} / 3$ recanalization (95\% CI $72 \%$ to $85 \%$ ), there was an $8 \%$ rate of symptomatic intracranial hemorrhage and a procedural complication rate of $11 \% .^{21}$

Even before the major stroke trials, controversy existed as to whether or not MT for anterior circulation ELVO was efficacious in patients aged 80 or older, largely due to poor outcomes in most cases (ie, 60-70\%) despite good recanalization rates $(\sim 80 \%) .^{23}{ }^{24}$ This is problematic since about one-third of ischemic strokes occur in patients of this age and are associated with particularly poor clinical outcomes. ${ }^{25}{ }^{26}$ In 2016 , the trial investigators collectively pooled the data into a collaboration from the MR CLEAN, ESCAPE, REVASCAT, SWIFT PRIME, and EXTEND-IA trials into HERMES and performed a metaanalysis to evaluate whether the benefit of MT persisted when applied to specific populations of patients that were relatively under-represented in the major trials. ${ }^{6}$ One of these populations included the elderly, as some of the trials excluded elderly patients (SWIFT PRIME excluded patients $>80$ years and REVASCAT excluded those $>85$ years), and the median age of subject in the trials when pooled was only 68 . The authors found that although older age correlated with worse functional outcomes via the modified Rankin Scale (mRS), there remained a significant and fixed difference between the outcomes of the intervention (MT) and the non-intervention groups. Since then, more studies have emerged that evaluate technical feasibility with MT in the elderly and clinical outcomes, with mixed results. In the DAWN (diffusion-weighted imaging (DWI) or CTP Assessment with Clinical Mismatch in the Triage of Wake-Up and Late Presenting Strokes Undergoing Neurointervention with Trevo) ${ }^{27}$ trial, the elderly were included but only with specific core infarct sizes and/or National Institutes of Health Stroke Scale (NIHSS) 
Table 1 Summary of the mechanical thrombectomy section of the American Heart Association/American Stroke Association 2019 update of the 2018 acute ischemic stroke management guidelines (modified from Powers et al $^{19}$ )

COR LOE

3.7.1 Concomitant with IV alteplase

1. Patients eligible for IV alteplase should receive IV alteplase even if MT is being considered

I A

2. In patients under consideration for MT, observation after IV alteplase to assess for clinical response should not be performed

III: harm B-R

3.7.2 Zero to six hours after last seen well

1. Patients should receive MT with a stent retriever if they meet all the following criteria: (1) prestroke mRS score of 0 to 1 ; (2) causative । occlusion of the ICA or MCA segment 1 (M1); (3) age $\geq 18$ years; (4) NIHSS score of $\geq 6$; (5) ASPECTS $\geq 6$; and (6) treatment can be initiated (groin puncture) within 6 hours of symptom onset

2. Direct aspiration thrombectomy as first-pass MT is recommended as non-inferior to stent retriever for patients who meet all the following criteria: (1) prestroke mRS score of 0 to 1 ; (2) causative occlusion of the ICA or M1; (3) age $\geq 18$ years; (4) NIHSS score of $\geq 6$; (5) ASPECTS $\geq 6$; and (6) treatment initiation (groin puncture) within 6 hours of symptom onset

3. Although benefits are uncertain, MT with stent retrievers may be reasonable for carefully selected patients with AIS in whom treatment IIb can be initiated (groin puncture) within 6 hours of symptom onset and who have causative occlusion of segment 2 (M2) or segment 3 (M3) of the MCAs

4. Although benefits are uncertain, use of MT with stent retrievers may be reasonable for patients with AIS in whom treatment can be initiated (groin puncture) within 6 hours of symptom onset and who have prestroke mRS score $>1$, ASPECTS $<6$, or NIHSS score $<6$, and causative occlusion of the ICA or proximal MCA (M1)

5. Although the benefits are uncertain, the use of MT with stent retrievers may be reasonable for carefully selected patients with AIS in whom treatment can be initiated (groin puncture) within 6 hours of symptom onset and who have causative occlusion of the ACAs, vertebral arteries, basilar artery, or PCAs

\subsubsection{Six to 24 hours after last seen well}

1. In selected patients with AIS within 6 to 16 hours of last known normal who have ELVO in the anterior circulation and meet other DAWN or DEFUSE 3 eligibility criteria, MT is recommended

2. In selected patients with AIS within 16 to 24 hours of last known normal who have ELVO in the anterior circulation and meet other DAWN eligibility criteria, MT is reasonable

\subsubsection{Technique}

1. Use of stent retrievers is indicated in preference to the MERCI* device

Ilb $\quad$ B-R

\section{A}

$B-R$

OE

A

2. The technical goal of MT should be reperfusion to a mTICI grade $2 \mathrm{~b} / 3$ angiographic result to maximize the probability of a good functional clinical outcome

3. To ensure benefit, reperfusion to $\mathrm{mTICI}$ grade $2 \mathrm{~b} / 3$ should be achieved as early as possible

Illb C-LD

4. In the 6- to 24-hour MT window evaluation and treatment should proceed as rapidly as possible to ensure access to treatment for the greatest proportion of patients

5. It is reasonable to select an anesthetic technique during EVT for AIS based on individual patient risk factors, technical performance of Ila the procedure, and other clinical characteristics

6. The use of a proximal balloon guide catheter or a large-bore distal-access catheter, rather than a cervical guide catheter alone, in conjunction with stent retrievers may be beneficial

7. Treatment of tandem occlusions (both extracranial and intracranial) during MT may be reasonable

8. The safety and efficacy of IV glycoprotein IIb/llla inhibitors administered during endovascular stroke treatment are uncertain

9. Use of salvage technical adjuncts, including intra-arterial fibrinolysis, may be reasonable to achieve $\mathrm{mTICI}$ grade $2 \mathrm{~b} / 3$ angiographic results

\subsubsection{Blood pressure management}

1. In patients undergoing $\mathrm{MT}$, it is reasonable to maintain the $\mathrm{BP}$ at $\leq 180 / 105 \mathrm{~mm} \mathrm{Hg}$ during, and for 24 hours after, the procedure 2. In patients who undergo MT with successful reperfusion, it might be reasonable to maintain BP at a level $<180 / 105 \mathrm{~mm} \mathrm{Hg}$

*Mechanical Embolus Removal in Cerebral Ischemia (MERCI) device.

ACA, anterior cerebral artery; AIS, acute ischemic stroke; ASPECTS, Alberta Stroke Program Early CT Score; BP, blood pressure; COR, class of recommendation; ELVO, emergent large vessel occlusion; EVT, endovascular thrombectomy; ICA, internal carotid artery; LOE, level of evidence; MCA, middle cerebral artery; mRS, modified Rankin Scale; MT, mechanical thrombectomy; mTICI, modified Thrombolysis in Cerebral Infarction; NIHSS, National Institutes of Health Stroke Scale; PCA, posterior cerebral artery.

score, and there was no dedicated age analysis. In the DEFUSE-3 (The Endovascular Therapy Following Imaging Evaluation for Ischemic Stroke $)^{28}$ trial, patients aged $>80$ years were excluded.

Online supplemental table 1 displays the various studies published, in chronological order, examining the relationship between elderly age and outcomes in MT. We identified 22 studies in the advanced elderly (ie, $\geq 80$ years) and very old (ie, $\geq 90$ years), four of which were meta-analyses, and a majority of which were retrospective, single-center analyses
(10), with some multicenter studies (six), including two with national registry data. Half of the studies (11/22) performed an analysis dedicated to nonagenarians. The majority of relevant endpoints included 90-day mRS score, 90-day mortality, TICI score, and rates of symptomatic intracranial hemorrhage (sICH). A subgroup analysis of patients enrolled in MR CLEAN showed significant improvements in functional outcome with MT regardless of age or initial Alberta Stroke Program Early CT Score (ASPECTS). ${ }^{29}$ The collective HERMES data ${ }^{6}$ also showed 
significant improvements in outcomes with the addition of MT, despite the overall rate of good outcome being lower than for younger patients. Many single-center retrospective studies have shown the technical feasibility of MT in the elderly, good recanalization rates, but low rates of good clinical outcomes. 2530 Some studies (including one that combines octogenarians and nonagenarians), ${ }^{31}$ show comparable clinical outcomes to those of younger patients. ${ }^{32} 33$ A few meta-analyses have shown poor outcomes in comparison with those for younger patients. ${ }^{21} 3435$ Important to note, there are limited data ${ }^{63}$ comparing octogenarians receiving MT with those who did not receive MT as the comparison group, with a clear benefit in functional outcomes for the former.

Some studies (also listed in online supplemental table 1) have performed a dedicated analysis on patients who are 'very old' (ie, aged $\geq 90$ years). Multiple multicenter retrospective studies show that MT is feasible in this population, but with low rates of good outcomes. ${ }^{36-38}$ These data were revalidated with additional national registry data from Germany. ${ }^{39}$ Some data from singlecenter studies also show that MT in nonagenarians is feasible, but with low rates of good outcomes, ${ }^{40}$ and a particularly high rate of hemorrhagic complications. ${ }^{41}$ Some data from single centers show no difference between octogenarians and nonagenarians ${ }^{42}$ or younger patients, ${ }^{43}$ and no difference in discharge disposition between younger patients ( $\leq 69$ years) and nonangenarians. ${ }^{44}$ There has been one meta-analysis for this topic showing high revascularization rates in nonagenarians with low rates of good clinical outcomes. ${ }^{45}$

\section{Summary of data}

- MT in octogenarians and nonagenarians is technically feasible with acceptable recanalization rates.

- Clinical outcomes, including 90-day mRS score and 90-day mortality, are improved in the elderly who receive MT compared with those who do not. Clinical outcomes in this population are worse than in younger patients (ie, aged <80) undergoing MT. It is unclear whether clinical outcomes in the very old are comparable, worse, or better in comparison with either the elderly or younger patients.

- Symptomatic intracranial hemorrhage $(\mathrm{sICH})$ rates may be higher in elderly patients undergoing MT.

\section{Recommendation}

- MT should not be withheld from octogenarians and nonagenarians, but rather evaluated on a case-by-case basis. (class II $a-b$, level B-NR and C-LD)

\section{Patients with thrombocytopenia}

There is a paucity of data guiding decisions for MT in thrombocytopenic patients (SWIFT PRIME and EXTEND IA excluded patients with platelet counts $<100 \mathrm{~K} / \mu \mathrm{L}$, and DAWN and DEFUSE-3 exlucded those $<50 \mathrm{~K} / \mu \mathrm{L}$ ). This latter population is particularly vulnerable, since the American Heart Association and Eur0pean Stroke Organization guidelines from 2018 both suggest a platelet count of $<100 \mathrm{~K} / \mu \mathrm{L}$ to be a contraindication to IV tissue plasminogen activator $(\mathrm{tPa}) .{ }^{46}$

We identified three studies evaluating the impact of thrombocytopenia in MT, of which one was a retrospective review of prospectively collected trial data, and two were retrospective single-center analyses (online supplemental table 2). Nogueira et $a l^{47}$ analyzed data retrospectively from the prospectively conducted MERCI and Muti MERCI trials. The authors identified a total of 35 patients with abnormal bleeding profiles, including thrombocytopenia (though only six of these 35 patients constituted this category), who underwent MT and compared outcomes with results for a group of 270 patients with normal bleeding profiles who underwent MT. Rates of sICH were comparable between the two groups, though the latter group did have better clinical outcomes, probably due to worse pre-stroke functional status. Mönch et $a l^{48}$ performed a retrospective analysis specifically examining thrombocytopenia (defined here as $<150 \mathrm{~K} / \mu \mathrm{L}$ ). The authors also included patients who presented with normal platelet count but experience a drop in platelet count. There was no statistical difference in rates of sICH in any of the groups, though patients with a drop in platelet count had worse mortality and functional outcomes, and thrombocytopenic patients had higher mortality. Finally, Desai et $a l^{46}$ retrospectively reviewed patients and found largely no major differences in rates of sICH (ie, the authors used three separate definitions of sICH, and one did have statistically significant difference between groups), but worse functional outcomes and higher mortality in the thrombocytopenic group.

\section{Summary of data}

- MT in thrombocytopenic patients does not result in higher rates of sICH than in patients with normal platelets, though mortality is higher and clinical outcomes after treatment are worse, probably due to pre-existing morbidity.

\section{Recommendations}

- MT should not be withheld from thrombocytopenic patients. (class IIb, level C-LD).

- There is no absolute minimum platelet count that excludes patients from MT given the high morbidity of ELVO. The expert panel considers $<20 \mathrm{~K}$ very concerning for potential hemorrhagic conversion of infarction or procedural complication, and hematologic consultation to understand the underlying etiology of extreme thrombocytopenia is reasonable. Some experts also consider platelet transfusion to be reasonable in patients with very low platelet count. (class IIb, level C-EO).

\section{Pediatric patients with ELVO}

The incidence of AIS in children is low at approximately 2.3 per 100000 children $^{49}{ }^{50}$ but can be devastating with a very high morbidity of $70 \%$ and a mortality rate of $3-6 \% .{ }^{5152}$ While the cause of pediatric stroke is poorly understood, it can be divided into childhood/adolescent (non-neonatal) and neonatal age groups. In the childhood/adolescent age group, common etiologies include congenital heart disease, arterial dissection, and sickle cell disease. For example, the Canadian Pediatric Stroke Registry revealed cardiac disease to be the cause in $25 \%$ of cases of pediatric AIS. ${ }^{53}$ On the other hand, among cryptogenic strokes, a patent foramen ovale was found to be the cause in $40-50 \%$ cases as opposed to $10-27 \%$ in the general population. ${ }^{54}$ Less common causes include inflammatory vasculopathies, head and neck trauma, and prothrombotic disorders. ${ }^{55}$ However, in a large number of cases, the cause is can often not be identified. This has led to the diagnosis of idiopathic etiology of stroke of childhood. In childhood, arteriopathy may be due to spontaneous or traumatic dissections, or infectious etiology like varicella or moyamoya syndrome. Childhood prothrombotic disorders are broad and include antiphospholipid antibody, anticoagulant factor deficiencies, and hyperhomocysteinemia. In the neonatal population, the etiology is often more elusive but, in general, congenital cardiac anomalies and thrombophilia 
conditions presenting with stroke are more common than in the older child. Other factors in the neonatal age group includes maternal pregestational and gestational factors plus fetomaternal vertically transmitted infections. Among the above mentioned factors, the incidence of acute ischemic strokes due to ELVO is found in a small cohort of patients.

In a large retrospective cohort analysis, Mallick et al reviewed 107 pediatric patients and found that only three patients would have been eligible for hyperacute thrombolysis with IV tPA. ${ }^{56}$ This study and the slow recruitment of patients to the TIPS (Thrombolysis In Pediatric Stroke) trial demonstrates the degree of difficulty in achieving timely access to thrombolytic therapy in the pediatric patient group. Since 2015 the established benefit of MT in adults has shown tremendous improvement in neurological function in recanalized patients in real-world application of randomized trial results. For pediatric stroke, several case series have been published and reviewed recently, which provide an argument for the use of MT in select pediatric patients who present with ELVO who satisfy tissue viability based imaging criteria. $^{57-59}$

As has been mentioned before the morbidity burden in pediatric ischemic stroke is immense. The plasticity of the pediatric brain can hypothetically extend the time in which to help patients with ELVO. Ravindra et al demonstrate that in their retrospective analysis of five centers at which pediatric AIS was treated, the median time from last known normal was 380 min (late window) and good clinical outcome from recanalization of ELVO was seen in $80 \%$ of patients. ${ }^{59}$ In their study, eight patients were selected for MT based on perfusion imaging criteria. Zhou et al demonstrate that Solitaire stent-based MT is feasible and effective in pediatric ELVO, although in a small group of patients. ${ }^{58}$ Bhatia et al in their review advocate a prospective multinational registry to evaluate the role of MT in anterior and posterior circulation ELVO. ${ }^{57}$

As with the adult population, suitability for thrombectomy in pediatric patients is determined by both clinical and radiologic parameters. In general, unnecessary exposure of neonates and children under the age of 10 to ionizing radiation should be avoided when there is a viable alternative. Targeted, limited sequence MRI/MR angiography (MRA) protocols that are designed for rapid and accurate delineation of large vessel occlusion, infarct core, and penumbra are ideal in the pediatric population. Such rapid protocols may include time of flight-MRA, 3D T2, 3D fluid-attenuated inversion recovery (FLAIR), gradient echo, and DWI sequences, or a combination thereof, and permit acquisition times of less than 15 min on modern scanners. Non-contrast MRA based on time of flight vessel imaging is the preferred mode of imaging for evaluation of ELVO. The sensitivity and specificity of this technique for ELVO detection is well established in the adult population. ${ }^{60}$ If needed, both contrast and non-contrast techniques can be applied to enable MRI perfusion analysis of core and penumbra in the affected brain tissue. Arterial spin labeling is a promising non-contrast perfusion technique, which may prove useful in assessing tissue viability. ${ }^{61}$ However, perfusion sequences may also be unnecessary in some patients, as a reliable surrogate determination of infarct core versus penumbra may be inferred via mismatch between regional diffusion restriction and signal hyperintensity on DWI B-1000 and FLAIR sequences, respectively. In children age 10 years or more and adolescents, the established CT based imaging protocols used in adults are appropriate (non-contrast CT, CT angiography (CTA) neck and head with or without CT perfusion). MRI techniques in older children can be used when there is iodinated contrast allergy.
Pediatric stroke intervention poses access, device, and technique related challenges. Femoral access for intervention is necessary, though appropriate sheath size is dictated by the age of the patient, which roughly correlates to femoral artery diameter. In general, the femoral artery of neonates and children up to 12 months will accommodate a 4 French sheath. In older children and adolescents, a 6Fr sheath may be the limit. This access consideration restricts the choice of guide catheter, and at the time of writing removes the availability of a balloon guide catheter for sheath sizes less then 6Fr. Alternative approaches include the use of an aspiration/intermediate catheter in place of a dedicated guide, through which routine microcatheters and micro-guidewires may be used for navigation of the aspiration catheter or the delivery and deployment of stent retrievers.

The intracerebral vessels in children may be more fragile than those in the adult population and may be damaged by minimal manipulation with intraluminal devices. The force applied to the pediatric endothelium must be titrated according to the size of the child on the operating table and the size of the cervical and intracranial blood vessels. Aspiration catheter and/or stentriever selection should be appropriately calibrated to the target vessel size diameter. The larger $6 \mathrm{~mm}$ stentrievers should be reserved for larger and older children. While complete reperfusion (TICI $>3$ ) should remain the goal of any stroke intervention, a lower threshold for terminating the procedure should arguably be made when multiple passes or more distal catheter positions would be required to achieve such a target. Given the current device-related limitations, one strategy has been the use of intraarterial tPA at the site of the ELVO. Despite limited proof for this strategy in the adult literature, ${ }^{62}$ the technique has been used in the pediatric population. Although the efficacy of intra-arterial lytic remains unproved, it might represent a potential last resort technique.

Currently, MT employs fluoroscopy which involves the application of ionizing radiation. As with all medical imaging-guided interventions, care should be taken to ensure the lowest possible dose of radiation is used to achieve the intended purpose of the intervention, also known as the ALARA (As Low As Reasonably Achievable) principle. While this principle should be applied to patients of all age groups, children are thought to be at particular risk of dose-dependent and -independent radiation-related effects (deterministic and stochastic). The dose reduction strategies employed for other pediatric neurointerventions and body interventions should be applied equally in stroke treatment. ${ }^{63}$ Strategies applied equally to the adult and pediatric population include: limiting the use of magnification, routine use of collimation, low pulse rates for fluoroscopy, minimizing the patient to detector distance, minimizing non-essential oblique projections, shielding radiosensitive organs where possible, and eliminating unnecessary digital subtraction angiograms. These measures can all substantially reduce the radiation exposure to both the patient and operator during MT procedures.

Post-thrombectomy care is critical in maximizing the benefits of recanalized vessels to the patients for translation to improved neurological function. Postoperatively, patients should be admitted to a pediatric or neonatal intensive care unit (ICU), with close attention to blood pressure goals and to minimize possibilities of reperfusion hemorrhage following recanalization. After recovery in the ICU, attention should be on aggressive rehabilitation with dedicated goal-directed physical therapy. In recent times, advances have been made in the understanding of neuronal functional recovery and how that might be optimized further by use of artificial intelligence and novel applications of both virtual and augmented reality rehabilitation platforms. ${ }^{6465}$ 
Summary of data

- Data for MT vary by age in the pediatric population.

\section{Recommendations}

- Mechanical thrombectomy should not be withheld from neonates, infants, children, and adolescents with large vessel occlusive stroke but rather be evaluated on a case-by-case basis. (class II $a-b$, level B-NR and C-LD)

- MT in neonates and infants (less than 1 year of age) is technically feasible and acceptable to offer in selected patients (class IIb, level C-LD)

- MT in children (age 1-9 years) is technically feasible and acceptable to offer in selected patients (class IIb, level C-LD)

- MT in adolescents (age 10-19 years) is technically feasible and acceptable to offer in selected patients (class IIa, level $B-N R$ )

- Standard techniques for radiation dose minimization (ALARA) should be applied in diagnostic and therapeutic applications for pediatric stroke. (class I, level B-NR)

- Where possible, and determined by local availability, initial radiologic work up of stroke in neonates, infants, and children below the age of 10 years should involve non-ionizing radiation, specifically MRI. (class IIa, level $B-R$ )

\section{Patients with ELVO and collagen vascular disorders}

Collagen vascular disorders (CVD) make up a subset of the broader category of inherited disorders of connective tissue. These are mostly single-gene disorders that impair the structure and/or function of connective tissue. Although the most common sequelae of these disorders may not include neurologic manifestations, when they do occur these are most commonly neurovascular due to alterations in vascular connective tissue. Given the rarity of these disorders, no large sample studies have evaluated the occurrence and management of ischemic stroke in these populations; however, published case series and technical reports are numerous and can serve as a basis from which to draw general conclusions.

Classically recognized CVD associated with neurovascular manifestations include Ehlers-Danlos type, Marfan syndrome, pseudoxanthoma elasticum, Loeys-Dietz syndrome and neurofibromatosis, among others. Ehlers-Danlos syndrome is group of disorders with a variety of genetic defects and inheritance patterns. Vascular Ehlers-Danlos syndrome has an estimated prevalence of $0.2-1 / 100,00$ and is the subtype associated with the most neurovascular manifestations, including arterial dissections, carotid-cavernous fistulas, ischemic stroke, and intracranial aneurysms. The underlying pathology involves disruption in the assembly of type III collagen fibers. Marfan's disorder is another monogenic disorder with autosomal dominant inheritance. The disorder is characterized by a mutation in the fibrillin 1 gene and it has a prevalence of 1/50 000. The most common vascular complications associated with this disorder are aortic root disease and mitral valve prolapse. However, neurovascular complications, such as spinal cord ischemia, cervical artery dissection, and intracranial aneurysms, have also been reported. Pseudoxanthoma elasticum has a prevalence of approximately $1 / 100000$ with a varied underlying genetic etiology and inheritance pattern. The disorder is most commonly associated with cardiovascular and ocular sequelae. However, cervical artery dissection, intracranial aneurysms, and cerebral infarction secondary to premature stenosis/occlusion of carotid and vertebral arteries have been described. ${ }^{66-70}$
Patients with CVD are considered very high risk for endovascular procedures given the presumed increased risk of iatrogenic vessel dissection or rupture. Standard screening for intracranial aneurysms with diagnostic cerebral angiography is not generally recommended in these patients for these reasons. ${ }^{67}{ }^{68}$ Historically, some studies have even advocated that conventional angiography is absolutely contraindicated as a diagnostic tool in patients with vascular Ehlers-Danlos syndrome. ${ }^{67}$ Additionally, transvenous approaches for treatment of intracranial fistulas, when possible, have been recommended as there is less risk of arterial injury. ${ }^{71}$ In the setting of carotid and vertebral artery dissections, evaluations with non-invasive imaging and medical management are the recommended standards. Despite the high risk of arterial injury, some patients may be refractory to medical management, necessitating endovascular interventions. Given the rarity of these cases, there have been no technical guidelines published for these patients. However, numerous case studies describe transarterial approaches to the stenting of carotid artery dissections, treatment of carotid-cavernous fistulas, and treatment of intracranial aneurysms using a standard endovascular technique and guidelines. ${ }^{72-75}$

Acute ischemic stroke is rare in CVD, but most reports are associated with cervical carotid or vertebral artery dissection. Additionally, cases of cerebral infarction secondary to intracranial steno-occlusive disease, with a moyamoya pattern, have been reported in neurofibromatosis 1 and pseudoxanthoma elasticum as well. ${ }^{666769}$ There have been no reports in the literature however, of AIS secondary to thromboembolism and no studies evaluating the use of MT in this patient population. Given this lack of data, major guidelines cannot be made about the safety and efficacy of neuroendovascular procedures in this population. However, although there are some reports of successful treatment, and no absolute contraindication to endovascular intervention can be made at this time, careful clinical consideration must be made when dealing with these patients.

\section{Summary of data}

- MT in patients with CVD may carry increased procedural risks, but this varies by the specific underlying CVD.

\section{Recommendations}

- Potential benefits and risks of MT should be evaluated on a case-by-case basis in patients with collagen vascular diseases with reference to the specific underlying disease. (class IIbIII, level C-EO)

- Diagnostic catheter angiography in patients with CVD carries increased risks; non-invasive imaging should be used whenever possible. (class III:Harm, level C-EO)

\section{Large vessel occlusion in patients following surgery}

Acute ischemic stroke following surgery is a major cause of perioperative patient morbidity and mortality. The overall incidence of stroke is variable depending the type of procedure, with cardiothoracic surgeries having the highest risk.

One of the largest prospective institutional databases of nearly 6000 patients concluded that the average incidence of cerebrovascular ischemia was $2.48 \%$; ELVO occurred in $10.9 \%$ of those patients. ${ }^{76}$ Prolonged aortic cross-clamp and cardiac bypass times were both associated with higher risk of AIS and specifically, ELVO. This suggests a synergistic effect of cerebral hypoperfusion and cardioembolic events as the mechanism of ischemia. Other suggested mechanisms of stroke in these patients are aortic atherosclerosis, cerebral hypothermia, hyperglycemia, anemia, 
atrial fibrillation, and genetic predisposition. ${ }^{77}$ In addition, the type of cardiac procedure was closely related to the incidence of AIS, which increased along with the complexity of the case. Boeken et al showed that the incidence of AIS in coronary artery bypass grafting (CABG) was 1.7\%, CABG and valve replacement $3.3 \%$, and multiple valve replacements $6.7 \%{ }^{78}$ In a Denmark cohort study of 2455 patients who underwent a transcatheter aortic valve replacement (TAVR) procedure, the overall risk of AIS was highest in the early postoperative phase (0-90 days) but not in the later phase (90 days-5 years). ${ }^{79}$ Patients who received anticoagulation during the early phase had nearly half the incidence of cerebral ischemic events as non-anticoagulated patients $(1.2 \%$ vs $2.4 \%)$. Despite these data, the recommendation for prophylactic use of anticoagulants in these patients has yet to be determined. ${ }^{80}$

The presentation of patients with ELVO is variable, based on the timing of the ischemic event. A JAMA article of a large prospective study of CABG over three decades showed that the timing of the event was intraoperative in $40 \%$ of patients and postoperative, with a peak at 40 hours after surgery, in $60 \%{ }^{81}$ Patients who have an intraoperative incidence of AIS, are less likely to be diagnosed promptly because of the duration of the surgery and long emergence from anesthesia, which does not allow for accurate neurological evaluation. Therefore, urgent CT angiography and perfusion imaging is indicated, especially in the first group of patients. If the deficit is detected promptly in the postoperative phase, then a non-contrast CT head and $\mathrm{CT}$ angiography are adequate for evaluation, based on the latest AHA/ASA guidelines. A thorough neurological examination protocol in the recovery unit should be strongly considered in cardiothoracic patients, in order to prevent delays in diagnosis and subsequent treatment.

Although data are limited on patient outcomes following mechanical thrombectomy for ELVO in perioperative AIS, most studies suggest that acute stroke intervention has a trend for improved functional outcomes and lower mRS scores in 3 months. ${ }^{76}$ Therefore, these patients should be strongly considered for the expanded window of MT based on individualized perfusion imaging data.

Following the detection of ELVO and AIS, the patient should be promptly transferred to the angiography suite. An implementation of a AIS protocol in the hospital is of paramount importance for fast detection and patient transportation. As these patients may present with cardiovascular instability, the presence of the cardiothoracic team and cardiopulmonary equipment allows for safe transportation. In the angiography suite, a thorough patient examination and communication with the primary team are required prior to choosing the arterial puncture site. If the patient still has arterial access from the original surgery, then using that site may expedite revascularization time. If bilateral femoral cutdowns were performed, such as in TAVR procedures, then a transradial approach should be considered instead. Conversely, if a radial artery harvest was used, then a transfemoral approach may provide faster access times.

Intraoperative considerations in these critical patients include the ability to administer anticoagulation or thrombolytic agents. A thorough communication with the original surgical team is essential to make this decision. In general, patients who undergo an open thoracic approach are not good candidates for intravenous tPA, but they may safely receive intravenous anticoagulants or focal intra-arterial thrombolytic agents. ${ }^{82}$ However, endovascular procedures, such as TAVR, generally do not have contraindications for either thrombolytic or anticoagulation therapies. ${ }^{80}$ Furthermore, the consistency of the thrombus may be variable as non-traditional types, such as lipids from pericardial suction aspirate, muscle, and fat emboli, may be involved. ${ }^{77}$ These may have a harder consistency and require aspiration thrombectomy with large-bore aspiration catheters, as opposed to stent retrievers.

The postoperative care of cardiac patients following MT requires a comprehensive approach involving both cardiothoracic and neurological teams. In general, a cardiac care unit with neurological protocol should be strongly considered. There are limited data, and therefore no recommendation, for postoperative medical management with antiplatelet or anticoagulation agents, therefore physician discretion is strongly advised.

\section{Summary of data}

- MT in patients who have recently undergone surgery can be successful and is especially important to consider given that surgery is a contraindication to intravenous thrombolysis.

\section{Recommendations}

- MT should not be withheld from patients who have recently undergone surgery (class IIa, level C-LD)

\section{Endocarditis}

Predicated on the high risk of hemorrhagic transformation, the use of thrombolytic agents is generally accepted as contraindicated if the etiology of infective endocarditis is suspected, ${ }^{83} 84$ making MT the de facto only option for intervention. To establish the odds of a favorable outcome in the particular population, it is necessary to note that the rate of neurologic complications during hospitalizations for infective endocarditis is high and associated with an increased risk of death ${ }^{85}$ Large strokes, due to their association with ELVOs, the target population considered here, have been found to be independently associated with mortality compared with patients who had transient ischemic attacks or small strokes that remained clinically silent. ${ }^{86}$

D'Anna performed an extensive review of the literature using the PRISMA framework. His conclusions represent the currently highest quality data point. ${ }^{87} \mathrm{He}$ reports results of 30 patients reported in 19 published case series out of 431 screened records. Overall, MT was as effective as in patients presenting with ELVOs due to non-endocarditis etiologies. The median NIHSS score dropped from 15 before to 2.5 after the procedure. An intracranial hemorrhage occurred in $13.3 \%$ of patients, and the overall mortality at 90 days was $23.3 \%$. Functional independence with an mRS score of less than 2 was achieved in $46.7 \%$ of patients. ${ }^{87}$ Of the notable randomized trials that established the usefulness of MT for large vessel occlusion, the results of this analysis were worse than some, but not all, falling somewhere in the middle: EXTEND-IA SWIFT PRIME showed $71 \%$ and $60 \%$ good outcome, respectively, and MR CLEAN showed 33\%. ${ }^{134} \mathrm{~A}$ less rigorous review by Bolognese and coworkers found a similar number of favorable outcomes, defined here as mRS score $\leq 3$ in $69 \%$ of patients. ${ }^{88}$

MT in patients with ELVO presumed to be the result of infective endocarditis has not been formally studied with a randomized controlled trial. Such a trial seems unlikely given the agreed-upon unfavorable outcome of the use of thrombolytic agents. Therefore, MT appears to be a reasonable option, with the understanding that its general safety and efficacy have not been established with high-quality data. 
Summary of data

- MT in patients with endocarditis appears to carry similar risks and benefits to those in patients without endocarditis.

\section{Recommendations}

- MT should not be withheld from patients with endocarditis (class IIb, level C-LD)

\section{Pregnancy}

The incidence of stroke in pregnancy in the USA, Western and Asian countries ranges from 4.3 to 210 per 100000 gestations. ${ }^{89-91}$ Management of acute ischemic stroke due to large vessel occlusion in a pregnant patient is challenging, given the considerations for the developing fetus. In the largest study of stroke in pregnancy and the post partum period, Kittner et al found that the risk of stroke was greatest during the third trimester or puerperium, rather than throughout the entire 9 months of pregnancy. ${ }^{89}$ Although certain conditions have a particularly strong association with stroke in pregnancy (such as eclampsia), or with the post partum period (such as cerebral venous thrombosis), most cerebral infarctions are due to arterial occlusion. ${ }^{9192}$

Administration of thrombolytic agents in pregnancy for suspected stroke is a complex decision with limited evidence. Pregnancy was an exclusion criterion in all IV thrombolysis trials, and formal studies examining the effects of thrombolytic agents on the outcomes of human pregnancy do not exist. Alteplase (tPA) is far too large a molecule to cross the placenta, and there are no quality human or animal data documenting the teratogenicity of thrombolytic agents. ${ }^{2} 93$ More than 200 reports exist in the literature of pregnant patients who have received thrombolytic therapy for various indications. ${ }^{91} 93$ Gartman systematically reviewed reports of pregnant patients who received tPA for neurologic indications (ie, acute stroke, venous sinus thrombosis), and showed no maternal deaths, no major bleeding events, and three fetal deaths/spontaneous abortions (none of which were attributed to thrombolytic administration). Complication rates of tPA in pregnancy are comparable with those in non-pregnant patients, with overall low risks of maternal mortality (1\%), maternal hemorrhage (8\%), and fetal loss (6\%). ${ }^{2} 9193-95$ The current AHA/ASA guidelines recommend IV tPA administration be considered when the expected benefits of treating life-threatening or potentially debilitating stroke outweigh the risk of uterine bleeding. ${ }^{19}$

Pregnancy was an exclusion criterion in the pivotal EVT clinical trials, and thus data from controlled clinical trials do not exist. Emergent EVT on the pregnant woman, like any other, requires the use of sedation and/or analgesics, iodinated contrast, and ionizing radiation. Neurointerventionalists should have a low threshold for consulting an anesthesiologist, especially for more complex cases, agitated patients, and/or for highrisk pregnancies. ${ }^{96}$ Iodinated contrast is a category B medication, and animal studies have not reported teratogenic or mutagenic effects from exposure. Human studies have not demonstrated any inhibitory effect of free iodide on neonatal thyroid gland function..$^{9596}$

The National Council on Radiation Protection and Measurements states that radiation exposure less than $50 \mathrm{mGy}$ to the fetus is considered negligible in comparison with baseline risks for all developmental abnormalities, but this risk increases significantly when exposure exceeds 150 mGy. ${ }^{97}$ The most important prenatal risk and deterministic effects occur at 8 to 15 weeks after conception, when the sensitive cortical brain cells are exposed to radiation greater than the threshold of $300 \mathrm{mGy} .{ }^{96}$ EVT procedures can involve cumulative radiation doses of approximately $1600 \pm 500 \mathrm{mGy} .{ }^{98}$ However, all available evidence and expert opinion suggest the deterministic risks of radiation exposure should not be taken into consideration when deciding on whether to perform EVT. Notwithstanding, neurointerventionalists should fully use fluoroscopic radiation safety features as endorsed by the Society of Interventional Radiology ${ }^{99}$ and according to ALARA principles. An experienced interventionalist(rather than a trainee) should perform as primary operator when possible, again in support of reduced fluoroscopy times. Other strategies include reducing the rate of pulsed fluoroscopy, use of last image hold, limiting the beam on-time, minimizing angiography acquisitions, limiting the use of magnification, using tight collimation, raising the patient away from the X-ray tube, minimizing the image intensifierpatient distance, removing the grid, reducing the tube current, and limiting femoral angiography. ${ }^{99}$ Use of lead shielding does not provide significant protection, but may lend a positive psychological reassurance to patients and their families. ${ }^{96}$

The safety and feasibility of EVT of ELVO in pregnancy is based solely on single-center case reports and one small case series, for a total of 14 patients. ${ }^{95}$ When examining these historical cases, despite the procedural diversity, 93\% (13/14 subjects) achieved a TICI $2 \mathrm{~b}$ or higher grade of recanalization, and all patients with follow-up data had a mRS score of 0-2 at final follow-up. Delivery outcomes consisted of seven normal full-term deliveries, one spontaneous abortion, two fullterm Cesarean sections, one emergency Cesarean section with delivery of a healthy newborn, and three cases of unknown delivery status. No maternal or fetal deaths were reported. EVT is seemingly a safe and feasible procedure for treating AIS due to ELVO in pregnancy, and should be considered for patients presenting as such.

Doppler fetal monitoring should performed in the first trimester and beyond, immediately before and after the procedure, to ascertain the health of the fetus. ${ }^{96}$ In certain circumstances, such as high-risk pregnancy and higher levels of sedation/ analgesia, continuous fetal monitoring during the procedure may be appropriate. Postprocedurally, patients should receive an obstetrics consultation, and postprocedure fetal ultrasound is obtained in the first trimester to evaluate fetal viability. ${ }^{96}$

\section{Summary of data}

- MT in pregnant patients is technically feasible with acceptable rates of recanalization and maternal and fetal clinical outcomes.

\section{Recommendations}

- Endovascular thrombectomy is a safe and feasible procedure for treating AIS due to ELVO in pregnancy, and should be considered for patients in a similar manner as would be indicated in a non-pregnant patient. (class IIa, level C-LD)

- Fluoroscopic radiation safety features should be fully used as well as a low threshold for anesthesia and obstetrics consultation in pregnant patients. (class $I I b$, level $C-L D$ )

\section{CONCLUSIONS}

Further studies are needed in patients excluded from 'standard' thrombectomy and randomized trials. As technology and techniques for thrombectomy continue to develop, evaluating their application to diverse patient populations with a variety of features or conditions that have excluded them from earlier 
trials is essential for appropriate dissemination of care. Given the rarity of some of these conditions, large randomized controlled trials may be impossible. Pragmatic design of multicenter trials and registries can help us better understand treatment for these patients.

\section{Author affiliations}

${ }^{1}$ Department of Neurology and Neurosurgery, Westchester Medical Center, Valhalla, New York, USA

${ }^{2}$ Department of Neurosurgery, Geisinger Health System, Wilkes-Barre, Pennsylvania, USA

${ }^{3}$ Department of Neurosurgery \& Radiology, University of Miami Miller School of Medicine, Miami, Florida, USA

${ }^{4}$ Department of Radiology, University of Michigan Health System, Ann Arbor, Michigan, USA

${ }^{5}$ Department of Neurosurgery, Icahn School of Medicine at Mount Sinai, New York, New York, USA

${ }^{6}$ Department of Neurological Surgery, Thomas Jefferson University Hospital, Philadelphia, Pennsylvania, USA

${ }^{7}$ Department of Neurosurgery, Montefiore Hospital and Medical Center, Bronx, New York, USA

${ }^{8}$ Department of Neurological Surgery, University of Miami School of Medicine, Miami, Florida, USA

${ }^{9}$ Department of Neurological Surgery, University of Washington, Seattle, Washington, USA

${ }^{10}$ Department of Interventional Neuroradiology, Goodman Campbell Brain and Spine, Carmel, Indiana, USA

${ }^{11}$ Division of Neurosurgery, University of Connecticut, Farmington, Connecticut, USA

${ }^{12}$ Department of Radiology, UCSF, San Francisco, California, USA

Twitter Reade De Leacy @rdeleacymd and Isaac Josh Abecassis @drjoshabecassis

Collaborators SNIS Standards and Guidelines Committee Members:Fawaz Al-Mufti, Sameer Ansari, Ketan Bulsara, Reade De Leacy, Neil Haranhalli, Steven W. Hetts, Seon Kyu Lee, Charles J. Prestigiacomo, Clemens Schirmer SNIS Board of Directors Members: Felipe C. Albuquerque, Sameer Ansari, Adam S. Arthur, Blaise W. Baxter, Michael Chen, Guilherme Dabus, Johanna T. Fifi, Justin F. Fraser, Michael T. Froehler, Mahesh J. Jayaraman, Richard P. Kluznik, William J. Mack, James M. Milburn, J Mocco, Ansaar T. Rai, Adnan H. Siddiqui, Jenny Tsai.

Contributors All authors made substantial contribution to the conception, design, or analysis or the work presented; took part in drafting or revising the manuscript critically for intellectual content; reviewed the final version of the manuscript submitted for publication; and agree to be accountable for the work.

Funding It is funded solely from internal resources of the Society of Neurolnterventional Surgery. RMS research is supported by the NREF, Joe Niekro Foundation, Brain Aneurysm Foundation, Bee Foundation, and by National Institute of Health (R01NS111119-01A1) and (UL1TR002736, KL2TR002737) through the Miami Clinical and Translational Science Institute, from the National Center for Advancing Translational Sciences and the National Institute on Minority Health and Health Disparities. The contents of this manuscript are solely the responsibility of the authors and do not necessarily represent the official views of the NIH. SWH: research is supported by NIH (R01CA194533, R42265316, R01EB012031).

Disclaimer RMS has an unrestricted research grant from Medtronic and has consulting and teaching agreements with Penumbra, Abbott, Medtronic, InNeuroCo and Cerenovus. SIT's institution has received grants from Medtronic and Cerenovus. SWH has consulting agreements with Medtronic, Kaneka, Imperative, and Cerenovus. SWH's institution has contract and grant support from Siemens, Stryker Neurovascular, and Route 92. RDL is a social media editor for JNIS.

Competing interests None declared.

Patient consent for publication Not required.

Provenance and peer review Not commissioned; internally peer reviewed.

Supplemental material This content has been supplied by the author(s). It has not been vetted by BMJ Publishing Group Limited (BMJ) and may not have been peer-reviewed. Any opinions or recommendations discussed are solely those of the author(s) and are not endorsed by BMJ. BMJ disclaims all liability and responsibility arising from any reliance placed on the content. Where the content includes any translated material, BMJ does not warrant the accuracy and reliability of the translations (including but not limited to local regulations, clinical guidelines, terminology, drug names and drug dosages), and is not responsible for any error and/or omissions arising from translation and adaptation or otherwise.

ORCID iDs

Clemens M Schirmer http://orcid.org/0000-0003-1743-8781
Stavropoula I Tjoumakaris http://orcid.org/0000-0002-1054-9414

Steven W Hetts http://orcid.org/0000-0001-5885-7259

\section{REFERENCES}

1 Berkhemer OA, Fransen PSS, Beumer D, et al. A randomized trial of intraarterial treatment for acute ischemic stroke. N Eng/ J Med 2015;372:11-20.

2 Demchuk AM. Yes, intravenous thrombolysis should be administered in pregnancy when other clinical and imaging factors are favorable. Stroke 2013:44:864-5.

3 Saver JL, Goyal M, Bonafe A, et al. Stent-retriever thrombectomy after intravenous t-PA vs. t-PA alone in stroke. N Engl J Med 2015;372:2285-95.

4 Campbell BCV, Mitchell PJ, Kleinig TJ, et al. Endovascular therapy for ischemic stroke with perfusion-imaging selection. N Engl J Med 2015;372:1009-18.

5 Jovin TG, Chamorro A, Cobo E, et al. Thrombectomy within 8 hours after symptom onset in ischemic stroke. N Engl J Med 2015;372:2296-306.

6 Goyal M, Menon BK, van Zwam WH, et al. Endovascular thrombectomy after large-vessel ischaemic stroke: a meta-analysis of individual patient data from five randomised trials. Lancet 2016:387:1723-31.

7 Deb-Chatterji M, Pinnschmidt H, Flottmann F, et al. Stroke patients treated by thrombectomy in real life differ from cohorts of the clinical trials: a prospective observational study. BMC Neurol 2020;20:81.

8 Fraser JF, Arthur AS, Chen M, et al. Society of Neurolnterventional Surgery recommendations for the care of emergent neurointerventional patients in the setting of COVID-19. J Neurointerv Surg 2020;12:539-41.

9 Ospel JM, Almekhlafi MA, Menon BK, et al. Workflow patterns and potential for optimization in endovascular stroke treatment across the world: results from a multinational survey. J Neurointerv Surg 2020;12:1194-8.

10 Jayaraman MV, Hemendinger ML, Baird GL, et al. Field triage for endovascular stroke therapy: a population-based comparison. J Neurointerv Surg 2020;12:233-9.

11 Kayan Y, Meyers PM, Prestigiacomo CJ, et al. Current endovascular strategies for posterior circulation large vessel occlusion stroke: report of the Society of Neurolnterventional Surgery Standards and Guidelines Committee. J Neurointerv Surg 2019:11:1055-62.

12 Mokin M, Ansari SA, McTaggart RA, et al. Indications for thrombectomy in acute ischemic stroke from emergent large vessel occlusion (ELVO): report of the SNIS Standards and Guidelines Committee. J Neurointerv Surg 2019;11:215-20.

13 Pierot L, Jayaraman MV, Szikora I, et al. Standards of practice in acute ischemic stroke intervention: international recommendations. J Neurointerv Surg 2018;10:1121-6.

14 Gandhi CD, Al Mufti F, Singh IP, et al. Neuroendovascular management of emergent large vessel occlusion: update on the technical aspects and standards of practice by the Standards and Guidelines Committee of the Society of Neurolnterventional surgery. J Neurointerv Surg 2018;10:315-20.

15 Leslie-Mazwi T, Chen M, Yi J, et al. Post-thrombectomy management of the ELVO patient: guidelines from the Society of Neurolnterventional Surgery. J Neurointerv Surg 2017;9:1258-66.

16 Pride GL, Fraser JF, Gupta R, et al. Prehospital care delivery and triage of stroke with emergent large vessel occlusion (ELVO): report of the Standards and Guidelines Committee of the Society of Neurointerventional Surgery. J Neurointerv Surg 2017:9:802-12.

17 McTaggart RA, Ansari SA, Goyal M, et al. Initial hospital management of patients with emergent large vessel occlusion (ELVO): report of the Standards and Guidelines Committee of the Society of Neurolnterventional Surgery. J Neurointerv Surg 2017:9:316-23.

18 Jayaraman MV, Hussain MS, Abruzzo T, et al. Embolectomy for stroke with emergent large vessel occlusion (ELVO): report of the Standards and Guidelines Committee of the Society of Neurolnterventional Surgery. J Neurointerv Surg 2015;7:316-21.

19 Powers WJ, Rabinstein AA, Ackerson T, et al. Guidelines for the early management of patients with acute ischemic stroke: 2019 update to the 2018 guidelines for the early management of acute ischemic stroke: a guideline for healthcare professionals from the American Heart Association/American Stroke Association. Stroke 2019:50:e344-418.

20 The Centre for Evidence-Based Medicine. The Oxford 2011 levels of evidence, 2011 Available: http://www.cebm.net/index.aspx?o=5653

21 Hilditch CA, Nicholson P, Murad MH, et al. Endovascular management of acute stroke in the elderly: a systematic review and meta-analysis. AJNR Am J Neuroradiol 2018;39:887-91

22 Bracard S, Ducrocq X, Mas JL, et al. Mechanical thrombectomy after intravenous alteplase versus alteplase alone after stroke (THRACE): a randomised controlled trial. Lancet Neurol 2016:15:1138-47.

23 Kurre W, Aguilar-Pérez M, Niehaus L, et al. Predictors of outcome after mechanical thrombectomy for anterior circulation large vessel occlusion in patients aged $=80$ years. Cerebrovasc Dis 2013;36:430-6.

24 Singer OC, Haring H-P, Trenkler J, et al. Age dependency of successful recanalization in anterior circulation stroke: the ENDOSTROKE study. Cerebrovasc Dis 2013;36:437-45.

25 Majidi S, Lee J, Balushi AA, et al. Endovascular thrombectomy in octogenarians and nonagenarians with large vessel occlusion: technical aspects and clinical outcome. $J$ Stroke Cerebrovasc Dis 2020;29:105120. 
26 Smithard DG. Stroke in frail older people. Geriatrics 2017;2. doi:10.3390/ geriatrics2030024. [Epub ahead of print: 15 Jul 2017].

27 Nogueira RG, Jadhav AP, Haussen DC, et al. Thrombectomy 6 to 24 hours after stroke with a mismatch between deficit and infarct. N Engl J Med 2018;378:11-21.

28 Albers GW, Marks MP, Kemp S, et al. Thrombectomy for stroke at 6 to 16 hours with selection by perfusion imaging. N Engl J Med 2018;378:708-18.

29 Ospel J, Kappelhof M, Groot AE, et al. Combined effect of age and baseline Alberta Stroke Program Early Computed Tomography Score on post-thrombectomy clinical outcomes in the MR CLEAN registry. Stroke 2020;51:3742-5.

30 Parrilla G, Carreón E, Zamarro J, et al. Recanalization and mortality rates of thrombectomy with stent-retrievers in octogenarian patients with acute ischemic stroke. Cardiovasc Intervent Radiol 2015:38:288-94.

31 Kawabata Y, Nakajima N, Miyake $\mathrm{H}$, et al. Endovascular treatment of acute ischaemic stroke in octogenarians and nonagenarians compared with younger patients. Neuroradiol J 2019;32:303-8

32 Ahn Y, Kim SK, Baek BH, et al. Predictors of catastrophic outcome after endovascular thrombectomy in elderly patients with acute anterior circulation stroke. Korean J Radiol 2020;21:101-7.

33 Hwang K, Hwang G, Kwon O-K, et al. Endovascular treatment for acute ischemic stroke patients over 80 years of age. J Cerebrovasc Endovasc Neurosurg 2015;17:173-9.

34 Zhao W, Ma P, Zhang P, et al. Mechanical thrombectomy for acute ischemic stroke in octogenarians: a systematic review and meta-analysis. Front Neurol 2019;10:1355.

35 Duffis EJ, He W, Prestigiacomo CJ, et al. Endovascular treatment for acute ischemic stroke in octogenarians compared with younger patients: a meta-analysis. Int J Stroke 2014:9:308-12.

36 Meyer L, Alexandrou M, Leischner $\mathrm{H}$, et al. Mechanical thrombectomy in nonagenarians with acute ischemic stroke. J Neurointerv Surg 2019;11:1091-4.

37 Janssen $\mathrm{H}$, Nannoni S, Francois $\mathrm{O}$, et al. Multicenter, retrospective analysis of endovascular treatment for acute ischemic stroke in nonagenarians. J Stroke Cerebrovasc Dis 2020;29:104817

38 Khan MA, Baird GL, Miller D, et al. Endovascular treatment of acute ischemic stroke in nonagenarians compared with younger patients in a multicenter cohort. J Neurointerv Surg 2017;9:727-31.

39 Meyer L, Alexandrou M, Flottmann F, et al. Endovascular treatment of very elderly patients aged $\geq 90$ with acute ischemic stroke. J Am Heart Assoc 2020;9:e014447.

40 Möhlenbruch M, Pfaff J, Schönenberger S, et al. Endovascular stroke treatment of nonagenarians. AJNR Am J Neuroradiol 2017;38:299-303.

41 Sussman ES, Martin B, Mlynash M, et al. Thrombectomy for acute ischemic stroke in nonagenarians compared with octogenarians. J Neurointerv Surg 2020;12:266-70.

42 Pinto MM, Nunes AP, Alves $M$, et al. Mechanical thrombectomy in stroke in nonagenarians: useful or futile? J Stroke Cerebrovasc Dis 2020;29:105015.

43 Andrews CE, Mouchtouris N, Fitchett EM, et al. Revascularization and functional outcomes after mechanical thrombectomy for acute ischemic stroke in elderly patients. J Neurosurg 2019:132:1182-7.

44 Agarwal S, Huang J, Scher E, et al. Mechanical thrombectomy in nonagenarians: a propensity score matched analysis. J Stroke Cerebrovasc Dis 2020:29:104870.

45 Bai $X$, Zhang $X$, Zhang $Y$, et al. Mechanical thrombectomy in nonagenarians: a systematic review and meta-analysis. Trans/ Stroke Res 2021;12:394-405.

46 Desai SM, Rocha M, Starr M, et al. Laterality is an independent predictor of endovascular thrombectomy in patients with low National Institute of Health stroke scale. J Stroke Cerebrovasc Dis 2018;27:3172-6.

47 Nogueira RG, Smith WS, MERCI and Multi MERCI Writing Committee. Safety and efficacy of endovascular thrombectomy in patients with abnormal hemostasis: pooled analysis of the MERCI and multi MERCI trials. Stroke 2009;40:516-22.

48 Mönch S, Boeckh-Behrens T, Kreiser K, et al. Thrombocytopenia and declines in platelet counts: predictors of mortality and outcome after mechanical thrombectomy. J Neurol 2019:266:1588-95.

49 Fullerton HJ, Wu YW, Zhao S, et al. Risk of stroke in children: ethnic and gender disparities. Neurology 2003:61:189-94.

50 Mallick AA, Ganesan V, Kirkham FJ, et al. Childhood arterial ischaemic stroke incidence, presenting features, and risk factors: a prospective population-based study. Lancet Neurol 2014;13:35-43.

51 Ellis MJ, Amlie-Lefond C, Orbach DB. Endovascular therapy in children with acute ischemic stroke: review and recommendations. Neurology 2012;79:S158-64.

52 Engle R, Ellis C. Pediatric stroke in the U.S.: estimates from the kids' inpatient database. J Allied Health 2012;41:e63-7.

53 DeVeber G. Risk factors for childhood stroke: little folks have different strokes! Ann Neurol 2003;53:149-50

54 Ganesan V, Prengler M, McShane MA, et al. Investigation of risk factors in children with arterial ischemic stroke. Ann Neurol 2003;53:167-73.

55 Ganesan V, Prengler M, Wade A, et al. Clinical and radiological recurrence after childhood arterial ischemic stroke. Circulation 2006;114:2170-7.

56 Marecos C, Gunny R, Robinson R, et al. Are children with acute arterial ischaemic stroke eligible for hyperacute thrombolysis? A retrospective audit from a tertiary UK centre. Dev Med Child Neurol 2015;57:181-6.
57 Bhatia K, Kortman H, Blair C, et al. Mechanical thrombectomy in pediatric stroke: systematic review, individual patient data meta-analysis, and case series. J Neurosurg Pediatr 2019:1-14

58 Zhou B, Wang X-C, Xiang J-Y, et al. Mechanical thrombectomy using a Solitaire stent retriever in the treatment of pediatric acute ischemic stroke. J Neurosurg Pediatr 2019;23:363-8.

59 Ravindra VM, Alexander M, Taussky P, et al. Endovascular thrombectomy for pediatric acute ischemic stroke: a multi-institutional experience of technical and clinical outcomes. Neurosurgery 2020;88:46-54.

60 Boujan T, Neuberger U, Pfaff J, et al. Value of contrast-enhanced MRA versus time-offlight MRA in acute ischemic stroke MRI. AJNR Am J Neuroradiol 2018;39:1710-6.

61 Grade M, Hernandez Tamames JA, Pizzini FB, et al. A neuroradiologist's guide to arterial spin labeling MRI in clinical practice. Neuroradiology 2015;57:1181-202.

62 Furlan A, Higashida R, Wechsler L, et al. Intra-arterial prourokinase for acute ischemic stroke. The PROACT II study: a randomized controlled trial. Prolyse in Acute Cerebral Thromboembolism. JAMA 1999;282:2003-11

63 The Image Gently Alliance. Available: https://imagegently.org

64 Grefkes C, Fink GR. Recovery from stroke: current concepts and future perspectives. Neurol Res Pract 2020;2:17.

65 Mubin O, Alnajjar F, Jishtu N, et al. Exoskeletons with virtual reality, augmented reality, and gamification for stroke patients' rehabilitation: systematic review. JMIR Rehabil Assist Technol 2019:6:e12010.

66 Schievink WI, Michels VV, Piepgras DG. Neurovascular manifestations of heritable connective tissue disorders. A review. Stroke 1994;25:889-903.

67 Debette S, Germain DP. Neurologic manifestations of inherited disorders of connective tissue. Handb Clin Neurol 2014;119:565-76.

68 Kim ST, Brinjikji W, Kallmes DF. Prevalence of intracranial aneurysms in patients with connective tissue diseases: a retrospective study. AJNR Am J Neuroradiol 2016;37:1422-6.

69 Kim ST, Brinjikji W, Lanzino G, et al. Neurovascular manifestations of connective-tissue diseases: a review. Interv Neuroradiol 2016;22:624-37.

70 Adham S, Billon C, Legrand A, et al. Spontaneous cervical artery dissection in vascular Ehlers-Danlos syndrome: a cohort study. Stroke 2021;52:1628-35.

71 Chuman H, Trobe JD, Petty EM, et al. Spontaneous direct carotid-cavernous fistula in Ehlers-Danlos syndrome type IV: two case reports and a review of the literature. $J$ Neuroophthalmol 2002;22:75-81.

72 Ohshima T, Miyachi S, Isaji T, et al. Bilateral vertebral artery dissection and unilateral carotid artery dissection in case of Ehlers-Danlos syndrome type IV. World Neurosurg 2019:121:83-7.

73 Linfante I, Lin E, Knott E. Endovascular repair of direct carotid-cavernous fistula in Ehlers-Danlos type IV. Case Reports 2014;2014:bcr2013010990.

74 Khan A, Chaudhary N, Pandey AS, et al. Direct puncture of the highest cervical segment of the internal carotid artery for treatment of an iatrogenic carotid cavernous fistula in a patient with Ehlers-Danlos syndrome. J Neurointerv Surg 2012;4:e29.

75 Dohle C, Baehring JM. Multiple strokes and bilateral carotid dissections: a fulminant case of newly diagnosed Ehlers-Danlos syndrome type IV. J Neuro/ Sci 2012;318:168-70

76 Sheriff $\mathrm{F}$ Hirsch J, Shelton $\mathrm{K}$, et al. Large-vessel occlusion stroke after cardiothoracic surgery: expanding time windows offer new salvage opportunities. J Thorac Cardiovasc Surg 2019:158:186-96. e2.

77 Hogue CW, Gottesman RF, Stearns J. Mechanisms of cerebral injury from cardiac surgery. Crit Care Clin 2008;24:83-98.

78 Boeken U, Litmathe J, Feindt P, et al. Neurological complications after cardiac surgery: risk factors and correlation to the surgical procedure. Thorac Cardiovasc Surg 2005:53:33-6.

79 De Backer O, Butt JH, Wong Y-H, et al. Early and late risk of ischemic stroke after TAVR as compared to a nationwide background population. Clin Res Cardiol 2020;109:791-801.

80 Mazer CD, Bhatt DL, Verma S. Anticoagulation following TAVR: no clear answer yet. Washington, DC: American College of Cardiology Foundation, 2019: 73. 22-8.

81 Tarakji KG, Sabik JF, Bhudia SK, et al. Temporal onset, risk factors, and outcomes associated with stroke after coronary artery bypass grafting. JAMA 2011;305:381-90

82 Watt TMF, Murray SL, Brescia AA, et al. Anticoagulation following mitral valve repair. $J$ Card Surg 2020:35:2887-94.

83 Masuda J, Yutani C, Waki R, et al. Histopathological analysis of the mechanisms of intracranial hemorrhage complicating infective endocarditis. Stroke 1992;23:843-50.

84 Demaerschalk BM, Kleindorfer DO, Adeoye OM, et al. Scientific rationale for the inclusion and exclusion criteria for intravenous alteplase in acute ischemic stroke: a statement for healthcare professionals from the American Heart Association/American Stroke Association. Stroke 2016:47:581-641.

85 Sonneville R, Mirabel M, Hajage D, et al. Neurologic complications and outcomes of infective endocarditis in critically ill patients: the ENDOcardite en reanimation prospective multicenter study. Crit Care Med 2011;39:1474-81.

86 Thuny F, Avierinos J-F, Tribouilloy C, et al. Impact of cerebrovascular complications on mortality and neurologic outcome during infective endocarditis: a prospective multicentre study. Eur Heart J 2007;28:1155-61.

87 D'Anna L. Endovascular treatment of ischemic large-vessel stroke due to infective endocarditis: case series and review of the literature. Neurol Sci 2020;41:3517-25 
88 Bolognese M, von Hessling A, Müller M. Successful thrombectomy in endocarditisrelated stroke: case report and review of the literature. Interv Neuroradiol 2018;24:529-32.

89 Kittner SJ, Stern BJ, Feeser BR, et al. Pregnancy and the risk of stroke. N Engl J Med 1996;335:768-74

90 Sharshar T, Lamy C, Mas JL. Incidence and causes of strokes associated with pregnancy and puerperium. A study in public hospitals of lle de France. Stroke in Pregnancy Study Group. Stroke 1995:26:930-6.

91 Treadwell SD, Thanvi B, Robinson TG. Stroke in pregnancy and the puerperium. Postgrad Med J 2008;84:238-45.

92 Pathan M, Kittner SJ. Pregnancy and stroke. Curr Neurol Neurosci Rep 2003;3:27-31.

93 Gartman EJ. The use of thrombolytic therapy in pregnancy. Obstet Med 2013:6:105-11.

94 Leonhardt G, Gaul C, Nietsch HH, et al. Thrombolytic therapy in pregnancy. J Thromb Thrombolysis 2006:21:271-6.
95 Limaye K, Van de Walle Jones A, Shaban A, et al. Endovascular management of acute large vessel occlusion stroke in pregnancy is safe and feasible. J Neurointerv Surg 2020;12:552-6.

96 Moon EK, Wang W, Newman JS, et al. Challenges in interventional radiology: the pregnant patient. Semin Intervent Radiol 2013;30:394-402.

97 Thabet A, Kalva SP, Liu B, et al. Interventional radiology in pregnancy complications: indications, technique, and methods for minimizing radiation exposure. Radiographics 2012:32:255-74

98 Cai $X$, Ding X, Wang W, et al. Radiation outcome in mechanical thrombectomy of acute ischemic stroke. Trans/ Neurosci 2019;10:10-13.

99 Dauer LT, Thornton RH, Miller DL, et al. Radiation management for interventions using fluoroscopic or computed tomographic guidance during pregnancy: a joint guideline of the Society of Interventional Radiology and the Cardiovascular and Interventional Radiological Society of Europe with endorsement by the Canadian Interventional Radiology Association. J Vasc Interv Radiol 2012;23:19-32. 